\title{
Cardiovascular functional disorder and stress among patients having neck-shoulder symptoms
}

Maarit Gockel, Harri Lindholm, Hannu Alaranta, Antti Viljanen, Ari Lindquist, Tuula Lindholm

\begin{abstract}
Objectives-To investigate if autonomic nervous system function, reflected in cardiovascular variables, among patients with neck-shoulder symptoms (tension neck group (T)) differed from that in a symptom free control group (C), and to establish its relation with pain and psychological stress.
\end{abstract}

Methods-Twelve women with tension neck and nine controls in secretarial jobs were studied. They underwent an orthostatic test, deep breathing test, Valsalva manoeuvre, isometric handgrip test, and muscular endurance test. Pain was measured using visual analogue scales, and psychological stress by the Modified Somatic Perception Questionnaire (MSPQ). Plasma endothelin-1 (ET-1) was measured using high pressure liquid chromatography and radioimmunoassay.

Results-Signs of psychological stress were significantly $(p<0.001)$ more common in group $T$ than in group C. Mean resting heart rate in group $T(\mathbf{7 7 . 8}$ (SE 2.9) beats/min; range 64-100) was significantly greater than that in group C (63.8 (3.1) beats/min; range $52-80)(p<0.01)$. In the orthostatic test, the overall changes in $\mathbf{R}-\mathbf{R}$ intervals during the first 40 heart beats after standing up and during seven minutes of testing differed significantly between the groups $(p<0.001,<0.05$, respectively). The increase in diastolic blood pressure in the three minute isometric handgrip test was significantly less in group $\mathrm{T}(19.4(3.5) \mathrm{mm} \mathrm{Hg}$; range -5 to 35) than in group C (30 (3.4) $\mathrm{mm} \mathrm{Hg}$; range $15-50)(p<0.05)$. The MSPQ score in the study group $(n=21)$ correlated positively with resting heart rate $(r=0.462$, $p<0.05)$ and negatively with increase in diastolic blood pressure $(r=-0.514$, $p<0.05$ ). Plasma concentrations of ET-1 did not differ between the groups.

Conclusion-Increased sympathetic activity was found among patients having neckshoulder symptoms. Local mechanisms may have influenced the cardiovascular changes observed during isometric testing in these patients.

(Ann Rheum Dis 1995; 54: 494-497)

The aetiology of musculoskeletal neck-shoulder symptoms has been connected with high perceived work load, monotonous work, and psychosocial stress factors, ${ }^{1}$ reduced local blood flow $^{2}$ and poor physical fitness. ${ }^{3}$

The autonomic nervous system also regulates local muscle blood supply; it is strongly modulated by emotional stress such as pain. ${ }^{4}$ In man, strong static or rhythmic muscular contraction normally causes an increased vasoconstrictive effect in peripheral vessels, as a result of which blood pressure increases. The vasoactive peptide, endothelin-1 (ET-1), is involved in baroreflex regulation during orthostatic stress. ${ }^{5}$

The purpose of this study was to evaluate autonomic nervous function in patients with tension neck problems. The results of standard cardiovascular tests were correlated to stress, pain, and the patient's functional status.

Materials and methods

One hundred women in secretarial jobs were asked about their neck and shoulder symptoms during the past year; 78 responded and were examined by a specialist in physical medicine and rehabilitation (MG).

Twelve women with symptoms of tension neck $^{6}$ during both the seven days preceding the reporting day and at least 30 days during the past year were selected randomly for the study group (tension neck group $(\mathrm{T})$ ). Clinical criteria for exclusion from the study were age over 50 years, some other explanation of pain (for example severe cervical arthrosis, humeral tendonitis), disease affecting autonomic nervous function (for example diabetes mellitus, hypertension, thyroid disturbances, anaemia), or regular medication.

Nine healthy women who had been free from symptoms during the preceding seven days and who had a maximum of seven days of symptoms of tension neck during the previous year were selected as the control group (C). Their mean age $(35 \cdot 1$ (SE 2.3) years, range 23-49) did not differ statistically significantly from that of group T (37.7 (2.4) years, range $25-44)$.

Pain was analysed by visual analogue scale. ${ }^{7}$ Psychological stress was assessed by the Modified Somatic Perception Questionnaire (MSPQ) developed by Main. ${ }^{8}$ Grip strength was measured with a Martin vigorimeter, and the muscular endurance of the upper limbs and dorsal spine (static arch-up) were evaluated according to Alaranta et al. ${ }^{9}$

Autonomic nervous system function was evaluated by standard cardiovascular tests. ${ }^{10}$ In the deep breathing test, the difference between the highest and lowest heart rates during one breathing cycle was calculated from the 
electrocardiogram; results were expressed as the mean of six consecutive cycles (beats $/ \mathrm{min}$ ). Traditionally, this test is considered to reflect the parasympathetic part of cardiovascular regulation. In the orthostatic test, the electrocardiogram, heart rate, blood pressure, and symptoms were recorded during active standing of eight minutes duration. Systolic and diastolic blood pressures and heart rate after one, three, five, and seven minutes standing were correlated against basal values after 10 minutes of supine rest. Heart rate variation during the first 40 seconds after active standing was recorded continuously; the relation of maximum heart rate to minimum heart rate during this period is considered to reflect, in some degree, parasympathetic regulation. The total results and individual crosssectional points during the first 40 seconds and during seven minutes were also compared between the groups. Increased resting heart rate is found in association with increased sympathetic activity. In the Valsalva test, the relation between maximum and minimum $R-R$ intervals after release of 15 seconds expiration against $15 \mathrm{~mm} \mathrm{Hg}$ of pressure was calculated; this test is considered a measure of parasympathetic cardiovascular regulation. In the isometric hand grip test, the subject adopted a sitting position, flexed the elbow $90^{\circ}$ and compressed a Martin vigorimeter for three minutes at $30 \%$ of maximal grip strength. The increase in diastolic blood pressure after three minutes of contraction was measured. This test mainly reflects sympathetic regulation of the peripheral vasoconstrictive system.

Plasma concentrations of the vasoactive peptide endothelin-1 (ET-1) were measured by a method comprising high pressure liquid chromatography and radioimmunoassay. ${ }^{11}$

STATISTICAL ANALYSIS

Differences and correlations were tested by Student's $t$ test and analyses of variance (solo Statistical system, 2.0, BMDP Statistical Software Inc). Values of $\mathrm{p}$ less than 0.05 were considered significant. Results are given as mean (SE).

Results

Visual analogue scores for pain were significantly greater in group $T$ (54.3 (5.8); range 20-92) than in group $C(0.7(0.5)$; range $0-4)$ $(p<0.001)$. The mean MSPQ score for psychological stress was also significantly greater in group T (9.75 (1.3); range 3-16)

Indices of autonomic nervous system function in the tension neck and control groups

\begin{tabular}{|c|c|c|c|}
\hline & $\begin{array}{l}\text { Tension } \\
\text { neck group }\end{array}$ & $\begin{array}{l}\text { Control } \\
\text { group }\end{array}$ & $p$ \\
\hline Resting heart rate (beats/min) & $\begin{array}{l}77 \cdot 8(2 \cdot 9) \\
{[64-100]}\end{array}$ & $\begin{array}{l}63 \cdot 8(3 \cdot 1) \\
{[52-80]}\end{array}$ & $<0.01$ \\
\hline Valsalva ratio & $\begin{array}{l}1 \cdot 72(0 \cdot 07) \\
{[1 \cdot 37-2 \cdot 39]}\end{array}$ & $\begin{array}{l}1 \cdot 77(0 \cdot 13) \\
{[1.35-2 \cdot 51]}\end{array}$ & \\
\hline $\begin{array}{l}\text { Hand grip test: } \\
\text { change in diastolic pressure after } 3 \mathrm{~min}(\mathrm{~mm} \mathrm{Hg})\end{array}$ & $\begin{array}{l}19 \cdot 4(3 \cdot 5) \\
{[-5 \text { to } 35]}\end{array}$ & $\begin{array}{c}30 \cdot 0(3 \cdot 4) \\
{[15-50]}\end{array}$ & $<0.05$ \\
\hline
\end{tabular}

Values are mean (SE) [range]. than in group $\mathrm{C}(1.7(0.3)$; range $0-3)$ $(\mathrm{p}<0.001)$.

Mean grip strength in group $\mathrm{T}(95$ (12) $\mathrm{kPa}$; range 35-150) did not differ significantly $(\mathrm{p}=0.09)$ from that in group $\mathrm{C}(127$ (12) $\mathrm{kPa}$; range $70-170)(p=0.09)$. The mean static endurance of the upper limbs in group $T$ (35 (3) seconds; range 20-60) was significantly less than that in group C (51 (3) seconds; range $40-65)(p<0.001)$, and in the static arch-up test of the dorsal spine, mean endurance was also less in group T (99 (22) seconds; range 23-310) than in group C (254 (50) seconds; range $55-480)(p<0.01)$.

There were no significant differences in resting systolic and diastolic blood pressures between the groups, but mean resting heart rate was significantly greater in group $T$ $(77.8(2.9)$ beats/min, range $64-100)$ than in group C (63.8 (3.1) beats/min, range 52-80) $(p<0.01)$ (table). In the orthostatic test, the mean ratio of maximum to minimum heart rates in group $T(1.5)$ did not differ significantly from that in group $C(1 \cdot 58)$.

Figure 1 shows the changes in R-R intervals over the first 40 heart beats after standing up. The intervals were significantly smaller in group $\mathrm{T}$ during the first 30 seconds after active standing up, but the parasympathetic braking effect seemed to be normal. The overall changes in $R-R$ intervals of the two groups differed significantly $(p<0.001)$ during the first 40 heart beats after standing up, this overall difference masking sequential changes in the difference between groups over the 40 beat profile: comparing beat for beat, from beats 1 to 8 the $p$ values for the significance of differences between the groups varied from 0.01 to 0.05 ; between beats 9 and 27 the range of $\mathrm{p}$ values was $0 \cdot 04-0 \cdot 11$; and beyond beat 27 the $p$ values were again less than 0.05 . The difference between the mean $R-R$ interval in group $T$ and that in group $C$, from beats 9 to 27 , was significant $(p<0.001)$.

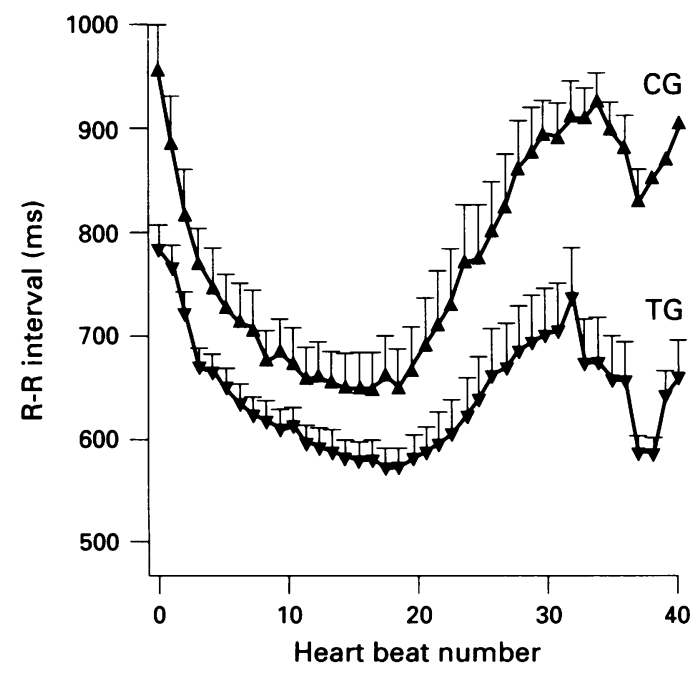

Figure 1 Mean (SE) changes in $R-R$ intervals during the first 40 heart beats after standing up in the tension neck group (TG: $\nabla)(n=12)$ and control group (CG: $\mathbf{\Delta})$ $(n=9)$. The overall results of the groups differ significantly ( $p<0.001$ ); paired beat by beat results: $p=0.01-0.05$ for beats 1 to $8, p=0.04-0.11$ for beats $9-27$ and $p<0.05$ from beat 28 . 


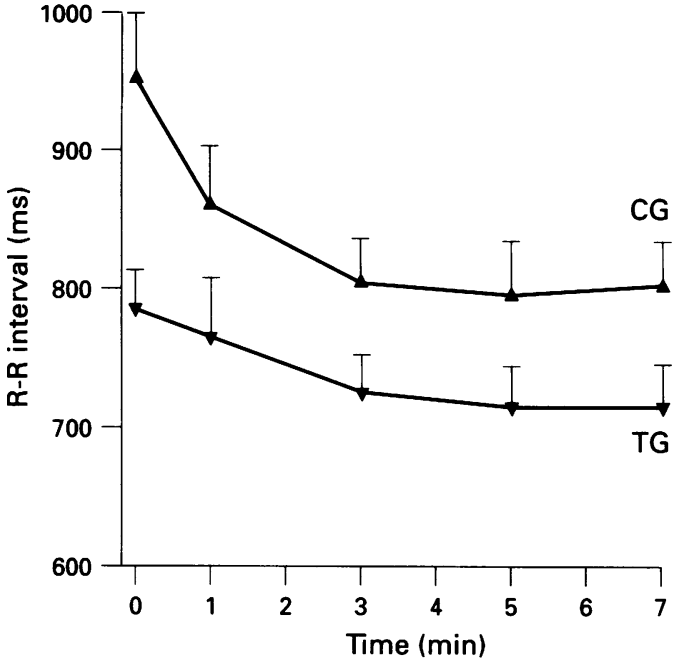

Figure 2 Mean (SE) changes in $R-R$ intervals during the seven minute orthostatic test in the tension neck group $(T G: \nabla)(n=12)$ and control group $(C G: \Delta)(n=9)$. Overall, the two groups differed significantly $(p<0.05)$ Minute by minute differences between groups at $0,1,3,5$ and 7 minutes have significance values of $p=0 \cdot 01-0 \cdot 12$.

Figure 2 shows the R-R intervals during the following seven minutes standing time: groups $T$ and $C$ differed significantly $(p<0.05)$. Comparing the groups minute for minute, the significance of the differences between them varied between $p=0 \cdot 01$ and $0 \cdot 12$.

The mean increase in diastolic blood pressure in the three minute handgrip test was $19.4(3.5) \mathrm{mm} \mathrm{Hg}$ (range -5 to 35 ) in group $\mathrm{T}$ and $30(3.4) \mathrm{mm} \mathrm{Hg}$ (range 15-50) in group $C(p<0.05)$. Heart rate indices in the deep breathing test of group $\mathrm{T}(21.8(1.7)$ beats/min; range $12-30$ ) and that of group $C$ $(20 \cdot 1(2 \cdot 4)$ beats/min; range $12-33)$ did not differ significantly. Similarly, there was no difference between the groups in Valsalva ratios (table).

The mean plasma endothelin-1 concentration was $5 \cdot 8(0.5) \mathrm{pg} / \mathrm{ml}$ (range $3 \cdot 6-9 \cdot 4$ ) in group $\mathrm{T}$ and $5.9(0.4) \mathrm{pg} / \mathrm{ml}$ (range $4 \cdot 2-8 \cdot 3$ ) in group $C$ (NS).

The MSPQ score in the study group as a whole $(n=21)$ correlated positively with resting heart rate $(r=0.462 ; p<0.05)$ and negatively with increase in diastolic blood pressure in the isometric hand grip test $(\mathrm{r}=-0.514 ; \mathrm{p}<0.05)$.

\section{Discussion}

The interaction between autonomic nervous system function and adaptation to pain is very complex. ${ }^{4}$ Also, peripheral responses are modulated by cortical activation or defence mechanisms activated by such influences as emotions. In this study, patients with tension neck had faster resting heart rates than the control group, which may have reflected increased sympathetic activity related to pain. This view is supported by other reports of increased sympathetic adaptation to pain. ${ }^{12}$ In addition, in the orthostatic test the overall changes in $\mathbf{R}-\mathrm{R}$ intervals during the first $\mathbf{4 0}$ heart beats and during the seven minutes following differed significantly between the patient and control groups. In the handgrip test, the diastolic blood pressure response in group $T$ was significantly attenuated in comparison with group C. Similar findings have been reported elsewhere, ${ }^{13}$ and increased sympathetic activity has been reported among young patients with chronic muscle pain. ${ }^{14}$ One explanation for our findings is that, in response to chronic neck pain, movement is restricted, and that as a result of this impairment in muscular work the afferent signal is diminished. The muscular endurance of the upper limbs was also weaker in group $T$, but maximal grip strength did not differ significantly between the patients and controls. Disturbances of parasympathetic regulation were not found. These results support the view that the moderate functional disorder observed in the tension neck group was attributable to increased sympathetic activity rather than decreased parasympathetic activity.

We conclude that increased sympathetic activity resulting from the stress of pain may play a role in maintaining symptoms in tension neck, but because our study was cross sectional, we were not able to establish a definitive cause and effect relationship.

Aerobic conditioning (fitness training) has been found to attenuate sympathetic activation ${ }^{15}$ and has been recommended for consideration as part of the treatment regimen for chronic muscle pain. While plasma endothelin-1 concentrations did not differ between our patient and control groups, thus excluding the peptide as a useful marker of changes in blood pressure regulation, we found that standard cardiovascular testing provided a convenient measure of increased sympathetic activity in patients with prolonged neck-shoulder symptoms. We wish to propose that such tests might usefully identify those patients who could benefit from aerobic fitness training to attenuate the sympathetic component of their pain.

This study was supported by the Scientific Fund of Instrumentarium and Neste Company.

1 Bongers $\mathrm{P} M$, de Winter $\mathrm{C} R$, Kompier $M$ A J, Hildebrandt $\mathrm{V}$ H. Psychosocial factors at work and musculoskeletal disease. Scand $f$ Work Environ Health 1993; 19: 297-312.

2 Larsson S-E, Bodegård L, Henriksson K G, Öberg Å P. Chronic trapezius myalgia. Acta Orthop Scand 1990; 61: 394-8.

3 Lehto T U, Helenius H Y M, Alaranta H. Musculoskeletal symptoms of dentists assessed by a multidisciplinary approach. Community Dent Oral Epidemiol 1991; 19: 38-44.

4 Stepherd R F J, Stepherd J T. Control of blood pressure and circulation in man. In: Bannister R, Mathias C J, eds. Autonomic failure. A textbook of clinical disorders of the autonomic nervous system 3 rd edn. Oxford: Oxford University Press, 1992; 78-93.

5 Vane J, Anggard E, Botting R. Regulatory functions of the vascular endothelium. N Engl f Med 1990; 323: 27-35.

6 Waris P, Kuorinka I, Kurppa K, et al. Epidemiologic screening of occupational neck and upper limb disorders. Methods and criteria. Scand $f$ Work Environ Health 1979; Methods

7 Huskisson E C. Measurement of pain. Lancet 1974; 2: 1127-31.

8 Main C J. The modified somatic perception questionnaire. F Psychosom Res 1983; 27: 503-14

9 Alaranta H, Soukka A, Harju R, Heliövaara M. Developing the techniques used for diagnosing musculoskeletal diseases. The measurement of the performance capacity of low back and neck in occupational health services. Helsinki: The Finnish Work Environment Fund A7, 1990 [in Finnish with English summary].

10 Piha S. Cardiovascular autonomic reflex tests. Normal responses and age-related reference values. Clin Phys 1991; 11: 277-90. 
11 Fyhrquist $F$, Metsärinne $\mathrm{K}$, Saijonmaa $\mathrm{O}$, Tikkanen $\mathrm{I}$, Rosenlöf $\mathrm{K}$, Tikkanen $\mathrm{T}$. Raised plasma endothelin-1 concentration following cold pressor test. Biochem Biophys Res Commun 1990; 169: 217-21.

12 Schott G D. Pain and the sympathetic nervous system. In: Bannister R, Mathias C J, eds. Autonomic Failure. $A$ textbook of clinical disorders of the autonomic nervous system, 3rd edn. Oxford: Oxford University Press, 1992; system, 3 rd
13 Pogaznick T, Sega S, Mesec A, Kiauta T. Autonomic function testing in patients with tension-type headache. Headache 1993; 33: 63-8.

14 Visuri $T$, Lindholm $H$, Lindqvist A, Dahlström $S$, Viljanen A. Cardiovascular functional disorder in primary fibromyalgia. Arthritis Care Res 1992; 5: 210-15.

15 Morgan B, Brammel H, Sable D. Effect of aerobic conditioning on cardiovascular response to isometric conditioning on cardiovascular response
exercise. $\mathcal{F}$ Appl Physiol 1982; 52: 1257-60. 